\title{
The Hidden Meaning of Planck's Constant
}

\author{
Robert L. Oldershaw \\ 12 Emily Lane, Amherst, MA 01002 USA \\ *Corresponding Author: rloldershaw@amherst.edu
}

Copyright (C) 2013 Horizon Research Publishing All rights reserved.

\begin{abstract}
A new cosmological paradigm based upon global discrete self-similarity proposes a radical revision in our understanding of atomic scale dynamics. The discrete fractal scaling of this paradigm predicts a very large and discrete scale-dependence for gravitational coupling constants. This alternative gravitational scaling leads to revised values for the Planck mass, Planck length and Planck time, which appear to be more unified and physically comprehensible than is the case with the conventional Planck scale values. The fundamental interrelationship of the revised Planck mass, the corrected gravitational constant for atomic scale systems, the reduced Planck's constant and the velocity of light suggests a hidden meaning for Planck's constant. Within the context of the discrete fractal cosmological paradigm, Planck's constant is revealed as the fundamental unit of gravitational action for atomic scale systems. Implications for atomic scale dynamics are briefly outlined.
\end{abstract}

Keywords Planck's Constant, Fractals, Cosmology, Discrete Self-similarity, Gravitation

\section{The Origin of Planck's Constant}

Planck's constant entered physics in 1900 as a result of Max Planck's attempts to provide a theoretical explanation for the empirically discovered laws of blackbody radiation. ${ }^{[1]}$ He found that Wien's heuristic approximation and existing observations could be reproduced if one adopted the concept that matter was a collection of discrete harmonic oscillators that obeyed an energy/frequency law of the form:

$$
\mathrm{E}=\mathrm{h} v
$$

for the emitted electromagnetic radiation. Since $h$ has the dimensions of $\mathrm{ML}^{2} / \mathrm{T}$ which are the dimensions of action, i.e., energy multiplied by time, it was natural to think of $h$ in terms of action principles. The implication of Planck's discovery of $h$ was that the action of atoms is quantized and that $\mathrm{h}$ represents the fundamental unit of action for discrete atomic scale systems. Planck's constant has become an integral component of modern atomic and subatomic physics, such that an understanding of the microcosm without it is virtually unthinkable. However, as pointed out by Peacock, ${ }^{[1]}$ to this day physicists really have not had a convincing explanation for why action in the microcosm is quantized, nor why $h$ has the specific quantitative value of $6.626 \times 10^{-27}$ erg sec. Here we will discuss the possibility that a discrete fractal approach to modeling nature may offer a unique and deeper understanding of Planck's constant.

\section{The Discrete Fractal Paradigm}

The discrete fractal paradigm focuses on nature's fundamental organizational principles and symmetries, and is referred to as the Self-Similar Cosmological Paradigm. ${ }^{[2]}$ It emphasizes nature's hierarchical organization of systems from the smallest observable subatomic particles to the largest observable superclusters of galaxies. The new fractal paradigm also highlights the fact that nature's global hierarchy is highly stratified into discrete Scales, of which we can currently observe the Atomic, Stellar and Galactic Scales. A third important principle of the fractal paradigm is that the cosmological Scales are rigorously self-similar, such that for each class of objects or phenomena on a given Scale there is analogous class of objects or phenomenon every other cosmological Scale. The self-similar analogues from different Scales have rigorously analogous morphologies, kinematics and dynamics. When the general self-similarity among the discrete Scales is exact, the paradigm is referred to as Discrete Scale Relativity ${ }^{[3]}$ and nature's global space-time geometry manifests a new universal symmetry principle: discrete scale invariance.

Based upon decades of studying the scaling relationships among analogue systems from the Atomic, Stellar and Galactic Scales, ${ }^{[2]}$ a close approximation to nature's actual Scale transformation equations for the length (L), time (T) and mass (M) parameters of analogue systems on neighboring cosmological Scales $\Psi$ and $\Psi-1$ are as follows.

$$
\begin{aligned}
& \mathrm{L}_{\Psi}=\Lambda \mathrm{L}_{\Psi-1} \\
& \mathrm{~T}_{\Psi}=\Lambda \mathrm{T}_{\Psi-1}
\end{aligned}
$$




$$
\mathrm{M}_{\Psi}=\Lambda^{\mathrm{D}} \mathrm{M}_{\Psi-1}
$$

The self-similar scaling constants $\Lambda$ and $\mathrm{D}$ have been determined empirically and are equal to $\cong 5.2 \times 10^{17}$ and $\cong$ 3.174, respectively. ${ }^{[2]}$ Different cosmological Scales are designated by the discrete index $\Psi(\equiv \ldots,-2,-1,0,1,2, \ldots)$ and the Stellar Scale is usually assigned $\Psi=0$.

\section{Revised Scaling For Gravitation}

Since the discrete fractal scaling applies to all dimensional parameters, the Scale transformation equations also apply to dimensional "constants." Given the dimensionality of the gravitational constant, $\mathrm{L}^{3} / \mathrm{MT}^{2}$, the discrete fractal paradigm proposes that the gravitational coupling constants $G_{\Psi}$ scale as follows. ${ }^{[3]}$

$$
\mathrm{G}_{\Psi}=\left[\Lambda^{1-\mathrm{D}}\right]^{\Psi} \mathrm{G}_{0}
$$

where $G_{0}$ is the conventional Newtonian gravitational constant. Therefore the Atomic Scale value $\mathrm{G}_{-1}$ is $\Lambda^{2.174}$ times $\mathrm{G}_{0}$ and equals $\cong 2.18 \times 10^{31} \mathrm{~cm}^{3} / \mathrm{g} \mathrm{sec}^{2}$. Now that we have the discrete fractal paradigm's prediction for the appropriate $G_{\Psi}$ value that applies within Atomic Scale systems, we can derive a revised Planck mass, length and time, and compare the revised Planck scale with the conventional Planck scale.

\section{A Revised Planck Scale}

In the early 1900s Max Planck realized that his newly discovered fundamental constant of the microcosm could be combined with the other known and apparently universal constants $\mathrm{G}$ and $\mathrm{c}$ to form a unique set of mass, length and time parameters that defined what has come to be known as the Planck scale. The Planck mass $(\mathfrak{A l l})$, the Planck length $(\mathfrak{l})$ and the Planck time $(\mathbb{C})$ are derived from the following relations.

$$
\begin{aligned}
& \mathfrak{A l}=(\hbar \mathrm{c} / \mathrm{G})^{1 / 2} \\
& \mathfrak{l}=\left(\hbar G / \mathrm{c}^{3}\right)^{1 / 2} \\
& \mathbb{T}=\left(\hbar \mathrm{G} / \mathrm{c}^{5}\right)^{1 / 2}
\end{aligned}
$$

The quantitative values for the conventional Planck scale parameters are listed in Table 1.

In the early 1900 s it was not entirely clear what the Planck scale parameters corresponded to in nature since there was nothing observed at these values of $\mathfrak{A l}, \mathfrak{l}$ and $\mathbb{T}$, and at that point no unambiguous theoretical interpretation was available. Since that time, a better theoretical understanding of the Planck scale has emerged: the Planck scale parameters define the scale at which gravitation must be included in the dynamic modeling of the microcosm, i.e., the scale at which General Relativity and Quantum Electrodynamics both play major roles in the dynamics of the microcosm.
Table 1. Conventional Planck Scale

\begin{tabular}{|c|c|c|}
\hline Parameter & Value & Counterpart in Nature \\
\hline $\mathrm{M}$ & $2.17 \times 10-5 \mathrm{~g}$ & None observed \\
\hline $\mathrm{R}$ & $1.62 \times 10-33 \mathrm{~cm}$ & $?$ \\
\hline $\mathrm{T}$ & $5.43 \times 10-44 \mathrm{sec}$ & $?$ \\
\hline
\end{tabular}

When $\mathrm{G}_{-1}$ is substituted for $\mathrm{G}$ in (6) - (8), as mandated by the discrete fractal paradigm, a radically different set of $\mathfrak{A l}$, $\mathfrak{l}$ and $\mathbb{C}$ values is generated. These revised Planck scale results are given in Table 2.

Table 2 Revised Planck Scale

\begin{tabular}{|c|c|c|}
\hline Parameter & Value & Counterpart in Nature \\
\hline $\mathrm{M}$ & $1.20 \times 10-24 \mathrm{~g}$ & $\sim$ proton mass \\
\hline $\mathrm{R}$ & $2.93 \times 10-14 \mathrm{~cm}$ & $\sim$ proton radius \\
\hline $\mathrm{T}$ & $9.81 \times 10-25 \mathrm{sec}$ & $\sim$ proton radius $/ \mathrm{c}$ \\
\hline
\end{tabular}

Whereas the conventional set of Planck scale values constitutes a seemingly random collection of numbers that do not appear to correspond to anything observed in nature, the revised set of Planck scale values derived from the discrete fractal paradigm are self-consistent and are firmly linked to the scale of nature's most fundamental baryon: the proton. The value of the revised Planck mass is $\approx 0.72$ times the mass of the proton, the revised Planck length is $\approx$ 0.4 times radius of the proton, and the revised Planck time is $\approx 0.4$ times the proton radius divided by the velocity of light.

\section{The Meaning of Planck's Constant}

In trying to understand the meaning of h, we focus on (6) and make the assumption that $\mathfrak{A l}$ is not merely an approximate scale parameter, but rather that it is a fundamental constant of Atomic Scale dynamics. Given this assumption, $\mathfrak{A l l}=\left(\hbar \mathrm{c} / \mathrm{G}_{-1}\right)^{1 / 2}$ is a much more rigorous interrelationship involving four of the fundamental Atomic Scale constants. We may rearrange (6) to give

$$
\hbar=\mathrm{G}_{-1} \mathfrak{A l}^{2} / \mathrm{c} \text {. }
$$

Equation (9) makes it explicit that $h$ is primarily associated with Atomic Scale gravitational interactions. Within the context of the discrete fractal paradigm, Planck's constant equals $2 \pi \mathrm{G}_{-1} \mathfrak{A t}^{2} / \mathrm{c}$ and is the fundamental unit of gravitational action for Atomic Scale systems. The concept 
that gravitational interactions dominate the dynamics within Atomic Scale systems is consistent with a recent potential advance in our understanding of the fine structure constant. ${ }^{[4]}$ Within the context of the discrete fractal paradigm, the fine structure constant is identified as the ratio of the strengths of the unit electromagnetic and gravitational interactions within Atomic Scale systems. Therefore within Atomic Scale systems gravitational interactions generally are stronger than electromagnetic interactions by a factor of $\alpha^{-1}$, or $\cong 137.036$.

Since all cosmological Scales are rigorously self-similar to one another, there must be a separate set of $\mathfrak{A t}_{\Psi}, \mathfrak{R}_{\Psi}$ and $\mathbb{C}_{\Psi}$ values for each cosmological Scale, and their respective values are governed by the discrete Scale transformations (2) - (4), when measured relative to some fixed set of dimensional units. ${ }^{[3]}$ These Planck scale sets define the "bottom", or the most fundamental unit level, of the baryonic subhierarchy that characterizes each cosmological Scale. When we substitute $\hbar=G_{-1} \mathfrak{A l}^{2} / \mathrm{c}$ into (7) we get:

$$
\mathfrak{l i}=\mathrm{G}_{-1} \mathfrak{A t} / \mathrm{c}^{2},
$$

which is highly reminiscent of the standard Schwarzschild radius ( $\mathrm{R}$ ) equation for a non-rotating, uncharged black hole, and differs from $\mathrm{R}$ only by a factor of 2 . This result is consistent with a recent finding that Atomic Scale hadrons, such as the proton and the alpha particle, can be modeled as Kerr-Newman or Schwarzschild black holes if $\mathrm{G}_{-1}$ is adopted as the appropriate gravitational coupling factor within hadrons. ${ }^{[5]}$ One can also substitute $\hbar=\mathrm{G}_{-1} \mathfrak{A l}^{2} / \mathrm{c}$ into (8) and generate a new expression for $\mathbb{C}$ :

$$
\mathbb{C}=\mathrm{G}_{-1} \mathfrak{A t} / \mathrm{c}^{3} .
$$

It is somewhat ironic to think that for over 100 years the ubiquitous presence of $\mathrm{h}$ and $\hbar$ in the equations that govern atomic and subatomic physics has been thinly veiling the dominant influence of Atomic Scale gravitational interactions throughout the microcosm, while common knowledge proclaimed that gravitational interactions played only a trivial role in atomic physics. In actuality, it appears that every time $\mathrm{h}$ or $\hbar$ is present in an Atomic Scale equation, we may replace it with $2 \pi \mathrm{G}_{-1} \mathfrak{A t l}^{2} / \mathrm{c}$ or $\mathrm{G}_{-1} \mathfrak{A t}^{2} / \mathrm{c}$ to reveal the true dominant influence of gravitation within the microcosm.

\section{Implications for Atomic Scale Dynamics}

There are an enormous number of fundamental and secondary technical details regarding the physics and mathematics of the discrete fractal paradigm that remain to be explored and resolved. Before our new understanding of Planck's constant can be fully implemented, a considerable amount of effort and insight must be applied to these technical issues. Here we must content ourselves with using the general principles of the discrete fractal paradigm and the results derived above to outline broadly the basic ways in which the discrete fractal paradigm might alter our understanding of Atomic Scale dynamics. Below is a listing of the most important implications of defining $h$ as the fundamental unit of gravitational action for Atomic Scale systems.

(a) Particles, Nucleons and Nuclei: If $\mathrm{G}_{-1}$ is the correct coupling factor for gravitational interactions within Atomic Scale systems, and $h$ is the fundamental unit of gravitational action in the microcosm, then subatomic particles must be modeled as ultracompact gravitational objects. Currently the best available approximations for these particles are probably the Kerr-Newman, Schwarzschild and Reissner-Nordstrom black hole solutions of General Relativity. Unbound electrons might best be approximated as nearly structureless singularities, due to their substantial spin but relatively low mass, whereas hadrons would have definite sizes on the order of their Schwarzschild radii. Presumably their radii would be more accurately determined via Kerr-Newman solutions which take charge, mass and rotational angular momentum into account. Intriguing similarities between the physical characteristics of subatomic particles and black holes have been pointed out by several authors, as noted in [5].

(b) Neutral and Partially Ionized Atoms: Inside atoms the gravitational interaction is about 137.036 times stronger than the electromagnetic interaction and therefore the dynamics within atoms is dominated by gravitation. Since the gravitational interactions among unbound particles, atoms and ions are 38 orders of magnitude weaker than their internal gravitational interactions, the overwhelmingly dominant interactions between unbound Atomic Scale systems are the familiar electromagnetic interactions.

(c) Atomic and Stellar Wavefunctions: Assuming the discrete fractal paradigm is basically correct, when a proton and an electron make the transition from separate unbound particles to a single bound hydrogen atom, the virtually singular electron must decompose into a fluid-like plasma composed of enormous numbers of $\Psi=-2$ Subquantum Scale particles of relatively infinitesimal size, charge and mass. ${ }^{[2]}$ Schrodinger's "probability density", or $\psi^{2}$, would have to be reinterpreted as the actual matter distribution $^{[6]}$ of the vast numbers of $\Psi=-2$ subquantum plasma particles. An atom in a very high Rydberg state would have a semiclassical electronic structure approximated by orbiting particle-like solutions $^{[7]}$ of the Schrodinger equation. Atoms in the ground state and low energy states would have more wave-like electronic structures with subquantum plasma distributions characterized by the more familiar wavefunction shapes: 
spheroidal, toroidal, bipolar, etc. A recent paper ${ }^{[8]}$ demonstrating a high degree of self-similarity between the masses, sizes, shapes and frequency spectra of RR Lyrae variable stars and the masses, sizes, shapes and frequency spectra of excited helium atoms undergoing single-level transitions between states with principal quantum numbers of 7-10 lends credence to the idea that the physics of Atomic Scale systems and their Stellar Scale analogues might be rigorously self-similar. If this is the case, then being able to study the physics of analogues on radically different spatial and temporal scales should be of great benefit in developing unified models for stellar and atomic systems.

(d) Quantum Mechanics for Atomic and Stellar Systems: A reinterpreted quantum mechanics wherein gravitation plays the dominant role for internal interactions, while electromagnetism plays the dominant role for external interactions among unbound systems, is conceivable. This unified reinterpretation of quantum mechanics would be equally applicable to Atomic Scale systems, Stellar Scale systems, Galactic Scale systems, or systems on any other cosmological Scale.

The comments in this section provide only a speculative sketch of the basic implications for the revised Atomic Scale dynamics suggested by our new understanding of Planck's constant. No doubt many years of effort by the physics community will be required in order to develop the general principles of the discrete fractal paradigm into a rigorous and unified analytical theory, combining General Relativity and Schrodinger's Wave Mechanics in a way that is consistent with Discrete Scale Relativity.

\section{Some Open Questions}

Finally, we will close the present discussion of Planck's constant and the new Atomic Scale dynamics proposed by the discrete fractal paradigm with several questions for future study.

(a) It is curious that $\mathrm{Al}$ is close to the mass of the proton, but less by a factor of about $(1 / 2 \pi)^{1 / 2}$. Possibly this small disparity is due to the fact that two crucial energy sources are neglected: internal spin and electromagnetic interactions. However, an even more interesting and compelling explanation has arisen. For ultracompact objects that can be modeled in terms of Kerr-Newman solutions of the Einstein-Maxwell equations, there is a crucial distinction between black hole solutions and naked singularity solutions. For black holes, $\mathrm{m}^{2} \geq \mathrm{a}^{2}+\mathrm{q}^{2}$ where $\mathrm{m}, \mathrm{a}(\equiv \mathrm{J} / \mathrm{m})$ and $\mathrm{q}$ are the geometrized mass, specific angular momentum and charge of the ultracompact object. Using $\mathrm{G}_{-1}$ as the correct gravitational coupling factor in the geometrizing process, we find the following values of $\left[\mathrm{m}^{2} / \mathrm{a}^{2}+\mathrm{q}^{2}\right]$ for the electron, Planck mass, proton, ${ }^{4} \mathrm{He}^{++}$and ${ }^{56} \mathrm{Fe}^{+26}: 4.36 \times 10^{-13}, 1.31,4.86,1060$ and 1057. These results yield two important implications. Firstly, the physical distinction between hadrons and leptons appears to be whether they satisfy the constraint $\mathrm{m}^{2} \geq \mathrm{a}^{2}+\mathrm{q}^{2}$ or the constraint $\mathrm{a}^{2}+\mathrm{q}^{2}>\mathrm{m}^{2}$, respectively. Secondly, it appears that the Planck mass is very close to the unique mass for which $\mathrm{m}^{2}=$ $\mathrm{a}^{2}+\mathrm{q}^{2}$ for Atomic Scale systems. Therefore $\mathfrak{A t}$ probably does not represent the mass of an actual particle in nature, but rather it probably represents the "tipping point" mass that defines the lepton/hadron, or horizon-free/horizon-possessing, boundary for ultracompacts on a given cosmological Scale.

(b) Can Schrodinger's $\psi^{2}$ be successfully reinterpreted as the density of Subquantum Scale plasma particles? Work along these lines was attempted by A. O. Barut. ${ }^{[6]}$ Perhaps the new ideas introduced by the discrete fractal paradigm will contribute to the conceptual and analytical development of this research effort.

(c) By what mechanism does an ultracompact object such as an unbound electron, which is virtually a naked singularity, decompose into wavefunction-like plasma shell comprised of myriad Subquantum Scale particles when the electron becomes bound to a nucleus?

(d) If the discrete fractal paradigm heralds a new unified physics for all cosmological Scales, what is the best analytical framework for this unification? Would a simple combination of General Relativity, Electromagnetism, Wave Mechanics and Discrete Scale Relativity be sufficient, or is some alternative framework required, such as a discrete 5-dimensional Kaluza-Klein approach with the $5^{\text {th }}$ dimension related to discrete scale? Another possible framework might be a 4-dimensional spacetime whose fundamental global geometry has discrete conformal symmetry.

Clearly much work remains to be done before the discrete fractal paradigm evolves from the conceptual, empirical and scaling foundations of natural philosophy to mature mathematical physics. The general paradigm itself is singularly testable. The definitive predictions by which the discrete fractal paradigm can be unambiguously tested concern the exact nature of the galactic dark matter. ${ }^{[9]}$ Preliminary empirical results from relentlessly negative exotic particle searches and positive microlensing observations appear to be quite encouraging. ${ }^{[10]}$ Fifteen definitive predictions of Discrete Scale Relativity, including the vindicated prediction of pulsar-planet systems, have been documented and are readily available. ${ }^{[1]}$ Published 
observational results that support those predictions are cited and discussed in that document.

\section{REFERENCES}

[1] K. A. Peacock, The Quantum Revolution - A Historical Perspective, Greenwood Press, Westport, CT and London, 2008.

[2] R. L. Oldershaw, The Self-Similar Cosmological Model: Introduction And Empirical Tests, International Journal of Theoretical Physics, Vol. 28, No. 6, 669-694 1989 and The Self-Similar Cosmological Model: Technical Details, Predictions, Unresolved Issues, International Journal of Theoretical Physics, Vol. 28, No. 12, 1503-1532, 1989; also see http://www.amherst.edu/ rloldershaw which is the most comprehensive resource for studying the SSCP.

[3] R. L. Oldershaw, Discrete Scale Relativity, Astrophysics and Space Science, Vol. 311, No. 4, 431-433, 2007 [DOI: 10.107/s10509-007-9557-x]; also available at http://arxiv.org as http://arxiv.org/abs/physics/0701132 .

[4] R. L. Oldershaw, Meaning of the Fine Structure the Constant, Fractals (submitted), 2013; available at http://arxiv.org as http://arxiv.org/abs/0708.3501 .
[5] R. L. Oldershaw, Hadrons As Kerr-Newman Black Holes, Journal of Cosmology, Vol. 6, 1361-1374, 2010; available at http://arxiv.org as http://arxiv.org/abs/astro-ph/0701006 .

[6] A. O. Barut, Combining relativity and quantum mechanics: Schrödinger's interpretation of $\psi$, Foundations of Physics, Vol. 18, No. 1, 95-105, 1988.

[7] M. Kalinski, J. H. Eberly, J. A. West and C. R. Stroud, Rutherford atom in quantum theory, Physical Review A, Vol. 67, 032503, 2003.

[8] R. L. Oldershaw, Discrete Self-Similarity Between RR Lyrae Stars And Singly-Excited Helium Atoms, Electronic Journal of Theoretical Physics, Vol. 5, No. 17, 207-214, 2008; available at www.ejtp.com, or at http://arxiv.org as http://arxiv.org/abs/astro-ph/0510147 .

[9] R. L. Oldershaw, The Self-Similar Cosmological Paradigm: A New Test And Two New Predictions, Astrophysical Journal, Vol. 322, No. 1, 34-36, 1987.

[10] R. L. Oldershaw, Mass Estimates For Galactic Dark Matter Objects As A Test Of A Fractal Cosmological Paradigm, Fractals, Vol. 10, No. 1, 27-38, 2002.

[11] R. L. Oldershaw http://www.academia.edu/2917630/Predictions_of_Discrete_ Scale_Relativity, 2013. 